\title{
Benchmark for the Determination of the Positronium Formation Fraction in Interstellar Media
}

\author{
Daniel G. Cocks (1D), Himanshu Chaudhary, and Joshua R. Machacek (10) \\ Research School of Physics and Engineering, Australian National University, Canberra, ACT 2601, Australia; daniel.cocks@anu.edu.au \\ Received 2019 February 28; revised 2019 May 1; accepted 2019 May 1; published 2019 June 20
}

\begin{abstract}
Relativistic positrons $(>1 \mathrm{MeV})$ are produced from many sources in the Galaxy. Observations of positrons rely on the characteristic gamma-rays emitted from their annihilation with an electron, and the dominant annihilation pathways are determined by low-energy $(<1 \mathrm{keV})$ processes. Thus, detailed information on the transport of positrons from high to low energies is required to accurately determine the propagation of positrons in the interstellar medium. This work considers model cross sections with variable parameters to provide a benchmark for several quantities, including the positronium formation fraction and time to reach subpositronium threshold energies. We simulate these processes using Monte Carlo codes within the cold collisional regime and carefully consider the energy sharing in ionization events that leads us to highlight the need for accurate ionization cross sections, which are differential in energy loss. We include tabulated values so that our benchmark values can be reproduced by other transport codes.
\end{abstract}

Key words: gamma rays: general - ISM: kinematics and dynamics

\section{Introduction}

Positrons are produced in interstellar media from various sources (Prantzos et al. 2011). As a positron is an antielectron, it can interact with an electron to annihilate producing a number of gamma-ray photons. Many sources of positrons have been studied (Prantzos et al. 2011; Crocker et al. 2017; Siegert et al. 2016; Higdon et al. 2009) and their transport through various types of interstellar media (ISM) has been investigated (Guessoum et al. 2005; Jean et al. 2009; Alexis et al. 2014; Panther et al. 2018a; Wallyn et al. 1994; Bussard \& Ramaty 1978; Brown et al. 1984). The effect of additional heavy atomic species in the ISM has also been considered (Panther et al. 2018b).

The dominant pathway for positron annihilation is through the formation of positronium $(\mathrm{Ps})$, a bound state of a positron and an electron. The threshold for the Ps formation process for any particular target is open for energies that are the Ps-binding energy $(6.8 \mathrm{eV})$ below the ionization process for the target. However, the cross section for Ps formation is significant only within a few $100 \mathrm{eV}$ of this threshold, which has been observed for a number of atomic and molecular targets (Machacek et al. 2016). Conversely, direct annihilation of the positron with a target is negligible at energies where the Ps channel is open $\left(\sigma_{\mathrm{ann}} / \sigma_{\mathrm{Ps}}<10^{-5}\right)$, and remains small even relative to elastic cross sections at room temperature. Hence, each positron has a high chance to be lost to annihilation via Ps formation but will persist for a much longer time if it can survive to sub-Ps energies. During this time, a sub-Ps positron may travel a greater distance as it is thermalizing. This can have dramatic implications for the prediction of the types and densities of positron sources in the galaxy.

Many studies have proposed models for the constituents of interstellar media and their interactions with positrons (see Prantzos et al. 2011; Martin et al. 2012; Churazov et al. 2011). These studies have calculated the "positronium formation fraction," $f_{\mathrm{Ps}}$, the percentage of positrons that form positronium instead of annihilating directly. These fractions are usually quite high and almost all studies have found $f_{\mathrm{Ps}}>90 \%$. This high value can be explained through the energy dependence of the collisional processes available to a positron, but the precise value of $f_{\mathrm{Ps}}$ is sensitive to the cross sections in the region of Ps formation. For example, in many positron trapping experiments, the excitation cross sections in $\mathrm{N}_{2}$ and $\mathrm{CF}_{4}$ can dominate over Ps formation and this leads to lower formation fractions (Marjanović et al. 2016; Danielson et al. 2015). Furthermore, high-energy inelastic processes can cause the positron to "jump" over the region of strong Ps formation. Hence, the representation of energy losses in simulation codes is important to obtain reliable Ps formation fractions.

Unfortunately, the detail necessary for accurate simulations is not often available in cross section sets. In particular, cross sections that are differential in both angle and energy-loss are sometimes required. An alternative is to explore a large range of qualitative behavior by using model cross sections incorporating tunable parameters. This has motivated the present benchmark model that serves two purposes. First, it allows us to investigate what variations can be expected for $f_{\mathrm{Ps}}$ from different physical processes and mixtures of species. Second, the benchmark model provides a detailed test for codes that model systems including Ps formation. While many codes exist, it is difficult to assess their accuracy except through quantitative comparisons with identical inputs.

The transport of positrons is also investigated in terrestrial laboratories and falls into the field of swarm transport analysis (Charlton 2009). Many benchmarks, for both positron and electron transport through gases, have been devised to validate codes and better understand the underlying physical processes. For example, it is known that thermalization of positrons does not necessarily result in a Maxwellian distribution (Marjanović et al. 2012) due to the "hole burning" that annihilation processes can cause. These benchmarks indicate that nonhydrodynamic effects are important to accurately model light particle transport. This has consequences for the modeling of positrons and secondary electrons in fluids (Marjanović et al. 2015). 
Although, many gaseous transport benchmarks exist, these are often focused on the asymptotic time regime (see Reid 1979; Ness \& Robson 1986; Raspopovic et al. 1999; Dujko et al. 2008, 2010; Boyle et al. 2017 and references therein), describing the transport quantities that approach constants or regular oscillatory values. In contrast, the quantities described here are highly dependent on the initial condition of the positron distribution, and we describe quantities that characterize the transient path of the positron from high to low energy. In particular, the Ps formation process is important for astronomical observations, and, for typical positron tracks, this process is only significant in the middle of the evolution of the positron ensemble.

We focus on the energy regime of positronium formation in neutral media. Hence, we include only collisional processes with neutral particles and neglect processes that can occur at energies above $\mathrm{MeV}$, e.g., bremsstrahlung, Compton scattering, and synchrotron radiation. As we do not consider an ionized medium, we do not introduce a space-charge distribution or consider Coulomb collisions. Furthermore, we restrict our focus to the energetics of the particle track, ignoring the spatial path it takes. This allows us to neglect much of the angle dependence of the cross sections, although we do consider the energy loss in a collision due to recoil. In particular, we focus on the differential energy loss for ionization processes, leaving the angular details of the positron-electron-ion relative coordinates unspecified.

\section{Benchmark Processes and Parameters}

The benchmarks we propose consist of following the timedependent transport properties of a dilute positron distribution released into a gas in the swarm approximation, i.e., the positrons are assumed to not interact with one another and the medium is assumed to be undisturbed by the positrons. The model for the gas is loosely based on a cold atomic hydrogen gas that we use as a surrogate for a cold neutral ISM phase.

The positron distribution is released at time $t=0$ with energies that are normally distributed about a mean of $10 \mathrm{keV}$ and with a standard deviation of $1 \mathrm{keV}$. This choice of distribution is motivated to avoid quasi-deterministic behavior that is expounded on in Section 3. These positrons then undergo a series of collisions with the medium of density $n_{0}$ and constituent mass $m_{0}=1 \mathrm{amu}$. To approximate the typical shapes of cross sections for these processes, we introduce two "surge" functions to mimic the known threshold- and asymptotic-behavior of real cross sections. The first surge function decays exponentially (Machacek et al. 2016) $S_{\exp }(\varepsilon$; $\left.\varepsilon_{\mathrm{th}}, p, \lambda\right)=S_{\exp }\left(x=\varepsilon-\varepsilon_{\mathrm{th}} ; p, \lambda\right)$, where

$$
S_{\exp }(x ; p, \lambda)= \begin{cases}0 & x<0, \\ \left(\frac{e}{\lambda}\right)^{p} x^{p} e^{-p x / \lambda} & x \geqslant 0,\end{cases}
$$

where $\lambda$ is a "stretch" parameter that locates the maximum value of 1 at $\varepsilon=\varepsilon_{\text {th }}+\lambda$, and $p$ is a parameter to control the exponential decay. The second surge function decays as a power law: $S_{\mathrm{pwr}}\left(\varepsilon ; \varepsilon_{\mathrm{th}}, p, \lambda\right)=S_{\mathrm{pwr}}\left(x=\varepsilon-\varepsilon_{\mathrm{th}} ; p, \lambda\right)$, where

$$
S_{\mathrm{pwr}}(x ; p, \lambda)= \begin{cases}0 & x<0, \\ \lambda^{p}(p+1)^{p+1} \frac{x}{(x+p \lambda)^{p+1}} & x \geqslant 0 .\end{cases}
$$

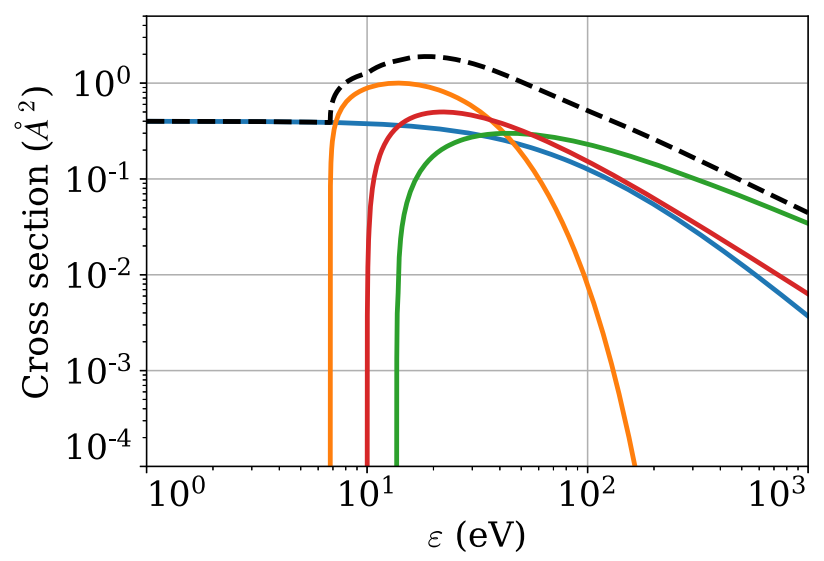

Figure 1. Cross sections for the default parameters of benchmark set B. The elastic (blue), Ps formation (orange), excitation (red), and ionization (green) cross sections are defined in the text with the parameters of $A_{\text {ion }}=0.3$, $\lambda_{\text {ion }}=30 \mathrm{eV}, A_{\text {exc }}=0.5, \varepsilon_{\text {exc }}=10 \mathrm{eV}$, and $\lambda_{\text {exc }}=12 \mathrm{eV}$. The total cross section is indicated as a black dashed line. The ionization cross section dominates for large energy. For benchmark sets A and C, the excitation cross section is either omitted or has a lower threshold, respectively.

This also has a maximum value of 1 at $\varepsilon=\varepsilon_{\text {th }}+\lambda$, and $p$ controls the power-law decay rate. Note that $S_{\mathrm{pwr}}\left(\varepsilon \rightarrow \infty ; \varepsilon_{\mathrm{th}}, \lambda, p\right) \propto \varepsilon^{-p}$.

The benchmarks are broken into three groups: A, B, and C; and each group includes elastic, Ps formation, and ionization processes. The elastic and Ps formation cross sections have a fixed shape, but the parameters of the ionization cross section are varied. Benchmarks $\mathrm{B}$ and $\mathrm{C}$ also include an excitation cross section of high and low threshold, respectively. Each process is described in more detail below, and an example cross-section set is shown in Figure 1.

\subsection{Ps Formation}

We fix the Ps formation cross section to be $\sigma_{\mathrm{Ps}}(\varepsilon)=A_{\mathrm{Ps}}$ $S_{\exp }\left(\varepsilon ; \varepsilon_{\mathrm{Ps}}, 0.5,7 \mathrm{eV}\right)$, where $\varepsilon_{\mathrm{Ps}}=\varepsilon_{\mathrm{IP}}-6.8 \mathrm{eV}, \varepsilon_{\mathrm{IP}}=$ $13.6 \mathrm{eV}$ is the ionization threshold, and $A_{\mathrm{Ps}}=1 \AA^{2}$. This is motivated by Machacek et al. (2016), where a comparison of cross sections for many atoms and molecules was made and such a fit was found to agree well with the majority of the species. ${ }^{1}$ The value of $A_{\mathrm{Ps}}$ acts as a comparative scale between the different cross sections.

\subsection{Elastic Collisions}

It is sufficient to only specify the momentum-transfer cross section of the elastic process as this accounts for the average energy loss in elastic collisions (Robson et al. 2017). We choose this cross section such that it is approximately constant for $\varepsilon=0$ and decays as $\varepsilon^{-2}$ for $\varepsilon \rightarrow \infty$, which follows the Born approximation (Landau \& Lifshitz 1981) at high energies. Our choice is a surge function with a negative threshold: $\sigma_{\mathrm{el}}^{\mathrm{mt}}(\varepsilon)=0.4 A_{\mathrm{Ps}} S_{\mathrm{pwr}}(\varepsilon ;-20 \mathrm{eV}, 2,20 \mathrm{eV})$.

\subsection{Ionization}

From the Born approximation (Landau \& Lifshitz 1981; Omidvar 1965; Gryziński 1965) it is known that the total ionization cross section decays as $\log \varepsilon / \varepsilon$ for high energies.

\footnotetext{
1 Note that we have modified the $p$ parameter of Machacek et al. (2016) from $p=0.45$ to $p=0.5$.
} 
Here, we capture the main feature by choosing a power-law surge function with $p=1$ for the ionization process: $\sigma_{\text {ion }}(\varepsilon)=A_{\text {ion }} A_{\mathrm{Ps}} S_{\mathrm{pwr}}\left(\varepsilon ; \varepsilon_{\mathrm{IP}}, 1, \lambda_{\mathrm{ion}}\right)$. This provides the dominant $1 / \varepsilon$ behavior for intermediate energies of the positron propagation. For this process we can vary two parameters: the magnitude, $A_{\text {ion }}$, and the stretch, $\lambda_{\text {ion }}$.

The energy lost by the positron in an ionization collision is $\varepsilon_{\text {IP }}$ plus that due to the recoil of the ion and electron. The recoil can be described as a sharing of the available kinetic energy post-collision between three particles. We use $\kappa$ to denote the "sharing fraction," i.e., the ratio of the positron's kinetic energy to the total kinetic energy of all particles, post-collision. A fraction, $1-\kappa$, is divided up among the ion and electron, although this division need not be specified. Explicitly, the energy after ionization is given by $\kappa \times\left(\varepsilon-\varepsilon_{\mathrm{IP}}\right)$

Note that recoil occurs in any collision, e.g., elastic collisions could be described in this notation with $1-\kappa \approx 2 m(1-$ $\cos \chi) / m_{0}$, where $\chi$ is the scattering angle. This amount is small due to the ratio $\mathrm{m} / \mathrm{m}_{0}$ between electrons and hydrogen, but can be significant at high energies, or accumulated over many collisions. In contrast, the light mass of an ionized electron allows for a larger amount of energy to be carried away from the positron in ionizing collisions leading to significant losses of energy in a single collision.

The value of $\kappa$ can be explicitly determined from conservation of energy and momentum if cross sections are used that are differential in all angles for the positron scattering and ejected angles for the ionized electron. However, as these differential cross sections are often unavailable, we instead treat the cross section as differential in $\kappa$ only, by defining a probability distribution $P(\varepsilon, \kappa)=\frac{d \sigma}{d \kappa} / \sigma(\varepsilon)$.

In the benchmark model considered here, we allow for two options for $P(\varepsilon, \kappa)$ : (a) a distribution given by the Born approximation result for hydrogen and (b) fixed sharing fractions. To implement (a) we make use of the distribution for K-shell ionization that has been calculated by Bethe (1930) and Mikhailov et al. (2008b) in the Born approximation. However, as these results are not in closed form and must be numerically integrated, we instead choose a simpler analytical fit. This is

$$
P_{K}(\varepsilon, \kappa)=\frac{1}{N(\varepsilon)}\left(\varepsilon(1-\kappa)+\frac{\varepsilon_{\text {th }}^{\text {ion }}}{2}\right)^{-2.1},
$$

where $N(\varepsilon)$ is a normalization factor. In contrast, the fixed sharing fraction, option (b), allows only a single $\kappa$ value, i.e., $P_{\tilde{\kappa}}(\varepsilon, \kappa)=\delta(\kappa-\tilde{\kappa})$. We will refer to this as the $\tilde{\kappa}$ distribution and use $\tilde{\kappa}=x$ as a shorthand for $P_{\tilde{\kappa}=x}(\varepsilon, \kappa)$.

\subsection{Excitation Collisions}

We include an excitation process in benchmarks B and C. This is chosen to be a surge function decaying as a power law, such that it is strong only close to the threshold: $\sigma_{\text {exc }}(\varepsilon)=$ $A_{\text {exc }} A_{\mathrm{Ps}} S_{\mathrm{pwr}}\left(\varepsilon ; \varepsilon_{\text {exc }}, 1.5, \lambda_{\text {exc }}\right)$. The value of $p=1.5$ was selected as a best-fit parameter for the shape of excitation cross sections in the literature (Kernoghan et al. 1996).

\subsection{Calculation Method}

We simulate the propagation of the positron distribution using two different particle-track Monte Carlo codes. These codes have been developed independently by us and provide a consistency check. One of these codes has been verified against several other benchmarks (Ness \& Robson 1986; Reid 1979; Tattersall et al. 2015) for steady-state transport of electrons in gases and liquids.

In short, the codes select a random initial energy for the positron from a normal distribution. During the propagation of the positron, it undergoes a series of collisions, determined by randomly sampling the time to collision (Tattersall et al. 2015) from the collision frequency $\nu(\varepsilon)=n_{0} v \sigma(\varepsilon)$. These collisions shift the positron toward lower energies, and the simulation is stopped when the positron either forms Ps or falls below the Ps threshold $\varepsilon_{\text {Ps. }}$.

In collisions other than Ps formation, we convert to the center of mass frame and resolve the collision. For elastic collisions we choose an isotropically random angle of scatter and then resolve the outgoing momenta and energies. As we use the elastic momentum-transfer cross section, this reproduces the average energy loss. For ionization or excitation collisions we subtract the threshold energy. In ionization collisions we additionally multiply the kinetic energy of the positron by the factor $\kappa$ sampled from $P(\varepsilon, \kappa)$, whereas for excitation collisions we resolve the collision with an isotropic scattering angle as is done for elastic collisions. Finally, we transform back to the ISM frame and continue until the next collision.

Note that we neglect the temperature of the gas, as this has a negligible effect at energies much greater than thermal for cold ISM phases. We also do not consider density variations, as for dilute gases the effect of density can be scaled out by defining the time and length scales to be $t^{*}=n_{0} t$ and $\boldsymbol{r}^{*}=n_{0} \boldsymbol{r}$, respectively (Robson et al. 2017).

\subsection{Quantities Compared}

The evolution of the distribution is characterized by a few key quantities that are of relevance to astrophysical studies. The first of these are the Ps formation fraction $f_{\mathrm{Ps}}$ and the survival positron fraction $f_{\text {surv }}=1-f_{\mathrm{Ps}}$, which is the fraction of positrons that reach sub-Ps energies and hence can later thermalize with the medium. To characterize the positrons that survive the Ps formation region, we consider a "low-energy reservoir" in which we place any positron that has reached subPs energies. This reservoir is described by an energy distribution, $f_{\text {res }}(\varepsilon)$, and the average time, $\tau_{\text {res }}^{*}$, that it takes for a positron to reach these energies. This provides a useful description for studies of positron propagation distance. Note that $\tau_{\text {res }}^{*}$ is scaled with density as mentioned in the previous section.

\section{Benchmarks}

Here, we present those cases where interesting variations in $f_{\text {surv }}$ occurred, or where we expected variations and they did not occur. In the Appendix we include a range of results that can be used to benchmark existing and new codes. However, for completeness we mention here that the parameters $\lambda_{\text {ion }}, \lambda_{\text {exc }}$, and $\varepsilon_{\text {exc }}$ had very little effect on $f_{\mathrm{Ps}}$, and only the choices of $A_{\text {ion }}$ and $P(\varepsilon, \kappa)$ caused any appreciable variation in $\tau_{\text {res }}^{*}$.

\subsection{Benchmark A (No Excitation)}

The first model we consider includes only the elastic, Ps formation and ionization processes with parameters that closely 

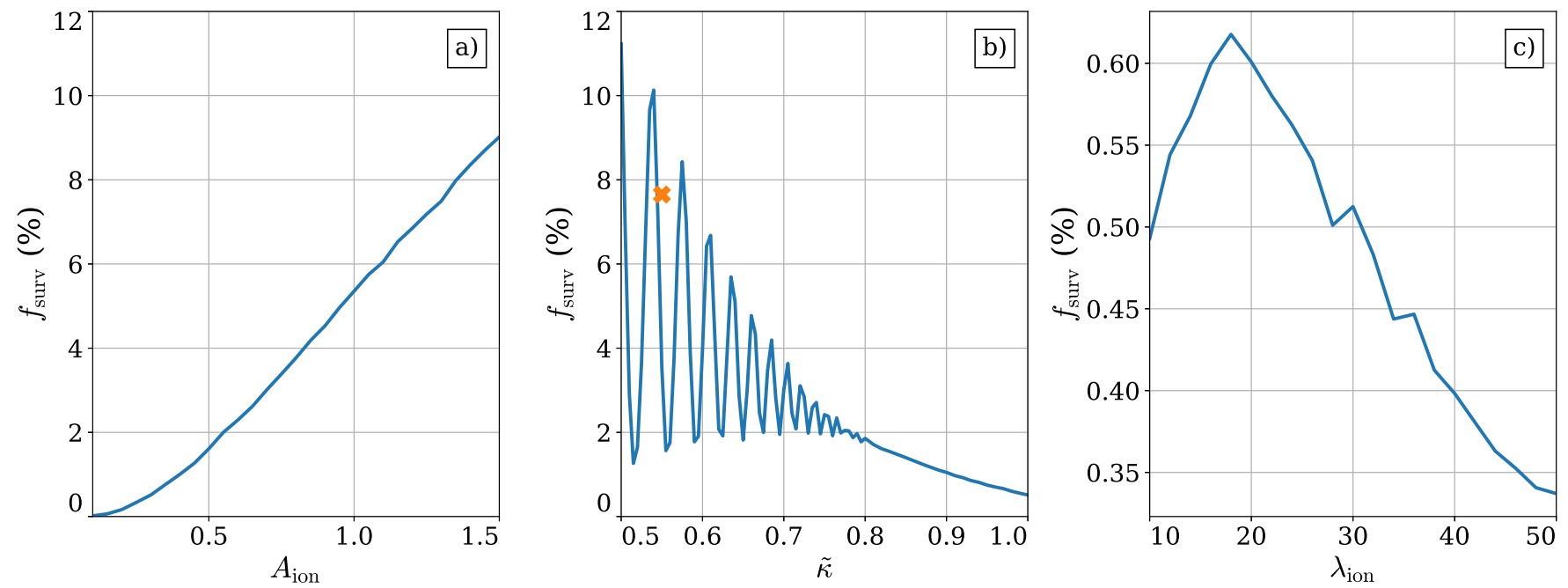

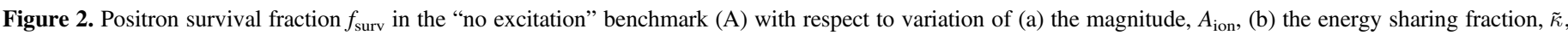

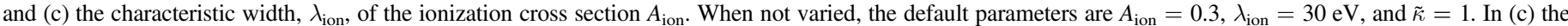
additional cross corresponds to a sharing distribution of $P_{K}$.

mimic the cross sections for atomic hydrogen:

$$
A_{\text {ion }}=0.3, \quad \lambda_{\text {ion }}=30 \mathrm{eV}, \quad \tilde{\kappa}=1 .
$$

We then vary one of the variables while keeping the others fixed. Note that using $\tilde{\kappa}=1$ implies that the positron retains all of the kinetic energy after an ionization collision. This is unphysical as we should expect the resulting ion and electron to recoil, otherwise they would not separate from one another. However, this behavior is sufficient to characterize the effect of large values of $\tilde{\kappa}$.

In Figure 2, we demonstrate the variation of $A_{\text {ion }}, \lambda_{\text {ion }}$, and $\tilde{\kappa}$. We can see that the strength of ionization is directly linked to the formation fraction. This is because an ionization event can allow the positron to "skip over" the Ps formation cross section and survive to sub-Ps energies. Surprisingly, we see that $\lambda_{\text {ion }}$ has only a moderate effect on this formation fraction, despite changing the location of the peak of $\sigma_{\text {ion }}$ by up to $3 \varepsilon_{\text {IP. }}$.

In contrast, the choice of sharing fraction $\tilde{\kappa}$ has a very large effect. For smaller values of $\tilde{\kappa}$, the curve oscillates widely. This is due to the absence of any randomness in the distribution $P_{\tilde{\kappa}}(\varepsilon, \kappa)$. If we neglect the small effect from elastic collisions, then any particular initial energy $\varepsilon_{0}$ will be deterministically connected to a set of lower energies $\left\{\varepsilon_{i}\right\}$ through the recursive relationship $\varepsilon_{i+1}=\tilde{\kappa} \times\left(\varepsilon_{i}-\varepsilon_{\mathrm{IP}}\right)$. Hence, a particular value of $\tilde{\kappa}$ can cause all $\left\{\varepsilon_{i}\right\}$ to lie outside of the region of strong Ps formation, leading to a high value of $f_{\text {surv }}$. However, the opposite can also occur, whereby the final ionization event lands the positron in the interval $\varepsilon_{\mathrm{Ps}}<\varepsilon_{i}<\varepsilon_{\mathrm{IP}}$, in which case the positron is almost certain to form Ps. ${ }^{2}$ We designate this behavior as "quasi-deterministic."

By including a distribution from which stochastic values of $\kappa$ can be randomly chosen, i.e., $P_{K}$, this issue of quasideterministic behavior is mitigated. At high energies, this

\footnotetext{
2 Without an excitation process, the positron can only survive to sub-Ps energies by a large number of elastic collisions in this interval between the Ps formation and ionization thresholds.

3 We aimed to mitigate this quasi-deterministic behavior by choosing an initial energy distribution with a large spread. However, the multiplicative nature of the energy sharing means that even a $1 \mathrm{keV}$ spread at high energies is only $10 \%$ of the mean energy, and it is this proportional spread that is maintained to low energies.
}

hydrogen-like description has a mean $\langle\kappa\rangle(\varepsilon)$ that is close to 1, but near to the ionization threshold, $\langle\kappa\rangle \approx 0.5$. From the resultant $f_{\text {surv }}$ calculated from the hydrogen-like description, shown as a cross in Figure 2(c), we find that this is close to what could be achieved with a fixed value of $\tilde{\kappa}=0.55$. We hence conclude that only the form of $P(\varepsilon, \kappa)$ in the energy regime close to threshold influences the Ps formation fraction.

\subsection{Benchmark B (High-energy Excitation)}

In this benchmark we test the inclusion of a single excitation process, with the parameters:

$$
A_{\text {exc }}=0.5, \quad \varepsilon_{\text {exc }}=10 \mathrm{eV}, \quad \lambda_{\text {exc }}=12 \mathrm{eV} .
$$

In particular, $\varepsilon_{\mathrm{Ps}}<\varepsilon_{\mathrm{exc}}<\varepsilon_{\mathrm{IP}}$, which provides an alternative route for positrons to avoid Ps formation. In addition to $\tilde{\kappa}=1$, we also consider the distribution $P_{K}$, to avoid the quasideterministic behavior identified in benchmark A.

In Figure 3 , we show the variation of $A_{\mathrm{exc}}, \varepsilon_{\mathrm{exc}}$, and $\lambda_{\mathrm{exc}}$. We can see an increase in the number of positrons reaching sub-Ps energies with larger $A_{\text {exc }}$ analogously to the increase observed with large $A_{\text {ion. }}$. As with the case of ionization, we also see only a small effect from modifying the maximum of the cross section through $\lambda_{\text {exc }}$. However, somewhat surprisingly, we see only a minor increase in the survival rate with an increase in the excitation threshold energy $\varepsilon_{\text {exc }}$ when $\kappa$ is given by $P_{K}(\varepsilon, \kappa)$. Our expectation was that a larger threshold would allow for the positron to more easily "skip over" the region of strong Ps formation, but this does not appear to be the case for the ordering $\varepsilon_{\mathrm{Ps}}<\varepsilon_{\mathrm{exc}}$.

\subsection{Benchmark C (Low-energy Excitation)}

In this benchmark set we also include a single excitation process, but with a low threshold:

$$
A_{\text {exc }}=1.0, \quad \varepsilon_{\text {exc }}=2 \mathrm{eV}, \quad \lambda_{\text {exc }}=10 \mathrm{eV} .
$$

This choice creates an ordering $\varepsilon_{\mathrm{exc}}<\varepsilon_{\mathrm{Ps}}<\varepsilon_{\mathrm{IP}}$. Compared with benchmark $\mathrm{B}$, there is a reduced amount of energy loss due to the smaller threshold energy of the process.

In Figure 4, we show the variation of $A_{\mathrm{exc}}, \varepsilon_{\mathrm{exc}}$, and $\lambda_{\mathrm{exc}}$. In contrast to benchmark $\mathrm{B}$, the variation of $\varepsilon_{\mathrm{exc}}$ in benchmark $\mathrm{C}$ 

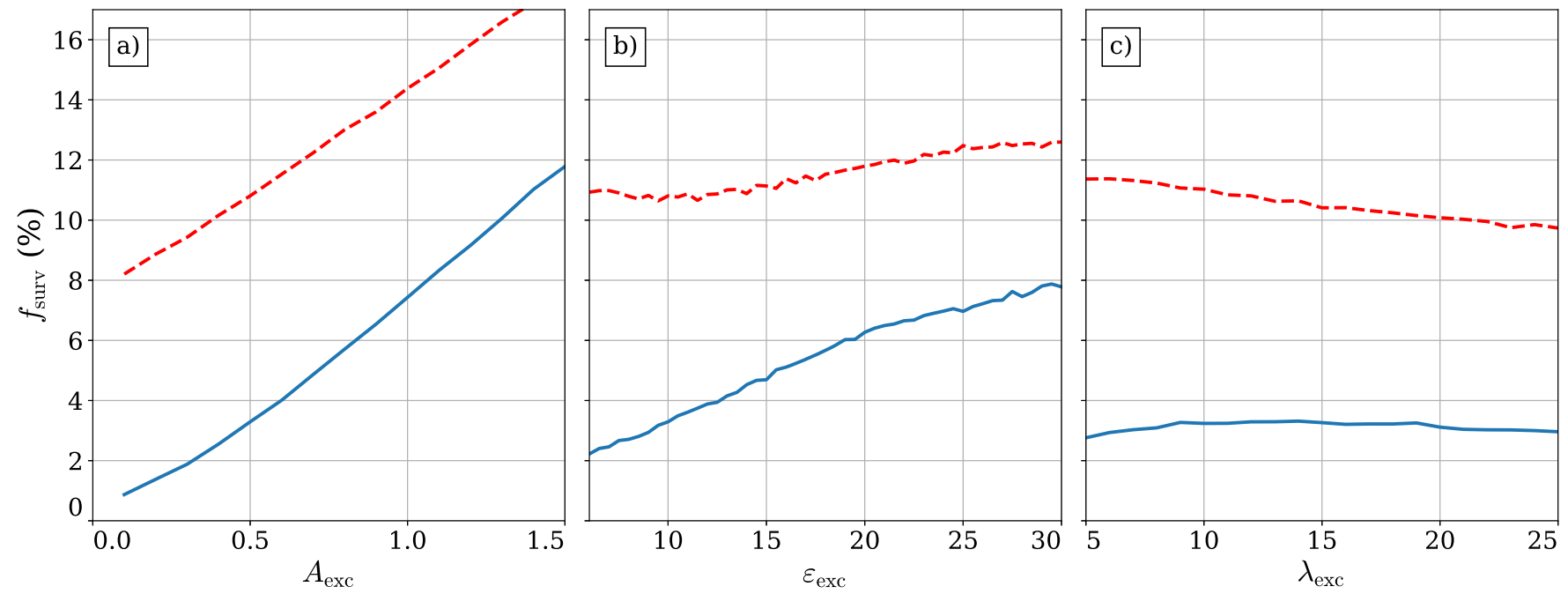

Figure 3. Positron survival fraction $f_{\text {surv }}$ in the "high-energy excitation" benchmark (B) with respect to variation of (a) the magnitude, $A_{\text {exc }}$, (b) the threshold energy, $\varepsilon_{\mathrm{exc}}$, and (c) the characteristic width, $\lambda_{\mathrm{exc}}$, of the excitation cross section. The blue solid lines correspond to default parameter values of $A_{\mathrm{exc}}=0.5, \varepsilon_{\mathrm{exc}}=10 \mathrm{eV}$, and $\lambda_{12} \mathrm{eV}$, with the remaining parameters as in Figure 2, i.e., a fixed energy sharing ratio of $\tilde{\kappa}=1$. The red dashed lines are identical, except that the energy sharing ratio corresponds to a hydrogen-like distribution.
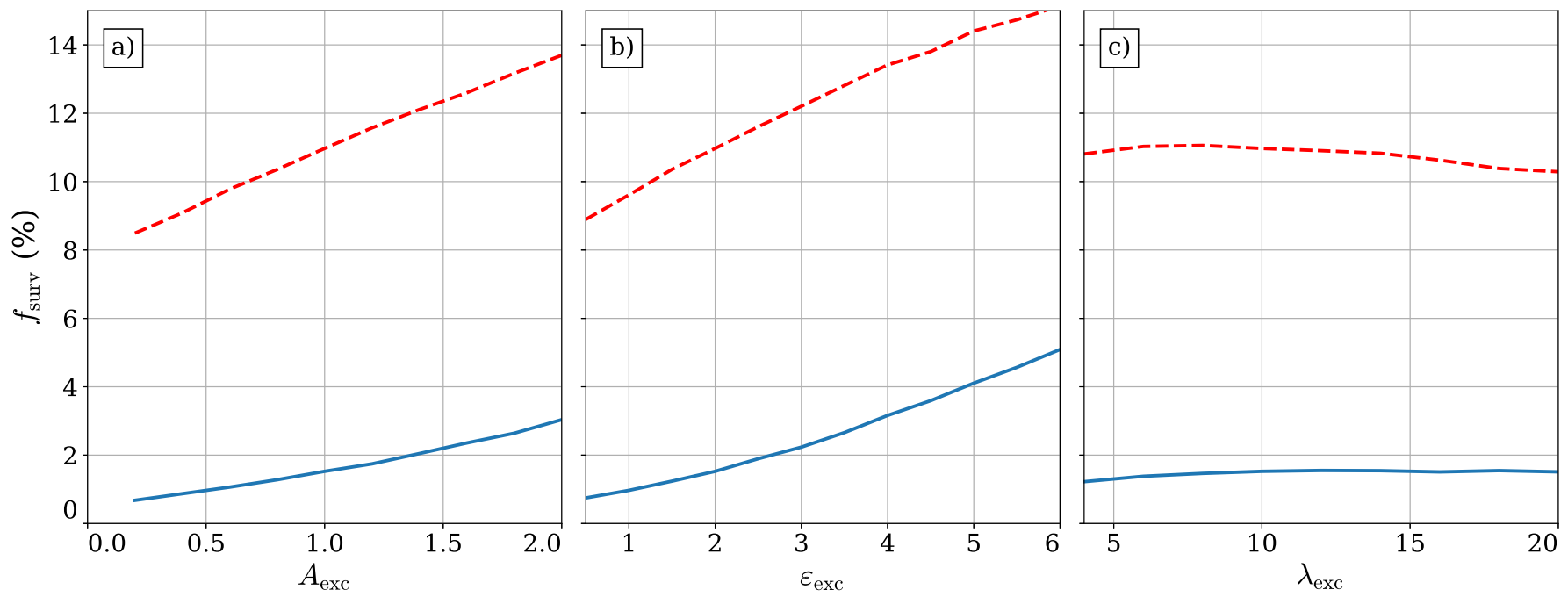

Figure 4. Positron survival fraction $f_{\text {surv }}$ in the "low-energy excitation" benchmark (C) with respect to variation of (a) the magnitude, $A_{\text {exc }}$, (b) the threshold energy, $\varepsilon_{\mathrm{exc}}$, and (c) the characteristic width, $\lambda_{\mathrm{exc}}$, of the excitation cross section. The default parameters are as in Figure 3 , but with $A_{\mathrm{exc}}=1.0 \mathrm{and} \varepsilon_{\mathrm{exc}}=2 \mathrm{eV}$.

gives rise to a more dramatic increase of the survival fraction. By the same reasoning, we expected that $f_{\text {surv }}$ would be sensitive to $\lambda_{\text {exc }}$, but instead we observe that the effect of varying $\lambda_{\text {exc }}$ is negligible.

\subsection{Low-energy Reservoir}

To finish, we return to benchmark $\mathrm{A}$ and focus on the parameters that characterize a low-energy reservoir, $\tau_{\text {res }}^{*}$ and $f_{\text {res }}(\varepsilon)$, the time and energy distribution, respectively, on first reaching sub-Ps energies. As we have previously discussed, quasi-deterministic behavior can arise with a choice of $\tilde{\kappa}=1$, so we also explore the behavior of the $P_{K}$ distribution.

In Figure 5, we can see that the time to reach the low-energy reservoir is strongly dependent on both $A_{\text {ion }}$ and $\tilde{\kappa}$. This is expected as lower values of $\tilde{\kappa}$ reduce the number of ionization collisions required to reach low energies and higher values of $A_{\text {ion }}$ decrease the average time between collisions. The $P_{K}$ distribution has a similar timescale as a $\tilde{\kappa} \approx 1$ distribution, in contrast to the $\tilde{\kappa} \approx 0.55$ behavior observed in $f_{\text {surv }}$ (see Figure 2(c)). This is because the majority of the collisions occur at high energies where $\langle\kappa\rangle(\varepsilon) \approx 1$ for $P(\kappa, \varepsilon)=P_{K}$.

Finally, in Figure 6 we show $f_{\text {res }}(\varepsilon)$. In both $\tilde{\kappa}=1$ and $P_{K}$, the energy distribution is somewhat linear; however, $P_{K}$ is much more uniformly distributed in energy.

\subsection{Sensitivity to Energy Sharing Distribution}

We want to highlight the behavior in the results due to the choice of the $P(\kappa, \varepsilon)$ distribution. We find the energy dependence of the energy sharing fraction in ionization with the choice of $P_{K}$ to be particularly interesting. Comparing the results to profiles of fixed $\tilde{\kappa}$, we found that the time to reach Ps formation energies for the distribution $P_{K}$ is similar to that for $\tilde{\kappa} \approx 1$, whereas the survival fraction of $P_{K}$ instead corresponds to $\tilde{\kappa} \approx 0.5$. By equating $\tilde{\kappa}$ to the distribution mean $\langle\kappa\rangle(\varepsilon)$ of $P_{K}$, we can identify that $\langle\kappa\rangle \approx 1$ occurs at high energies and $\langle\kappa\rangle \approx 0.5$ at energies close to threshold. Hence, the 

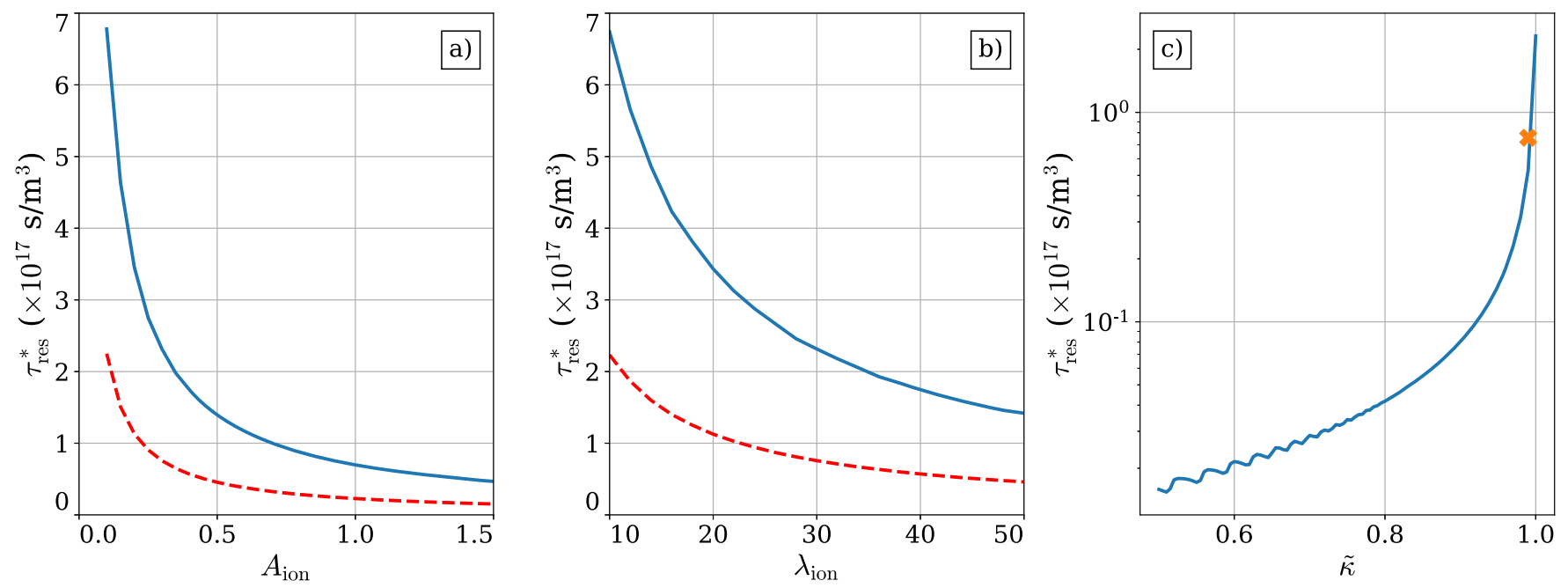

Figure 5. The scaled time, $\tau_{\text {res }}^{*}$, to "thermalization" in the "no excitation" benchmark with variation respect to (a) the magnitude, $A_{\text {ion }}$, (b) the characteristic width, $\lambda_{\text {ion }}$, and (c) the energy sharing fraction, $\tilde{\kappa}$, of the ionization cross section. The blue solid lines in (a) and (b) correspond to a single- $\kappa$ energy sharing distribution with $\tilde{\kappa}=1$, and the red dashed lines correspond to a hydrogen-like distribution. In (c) the blue line indicates a single- $\kappa$ energy sharing distribution with varying $\tilde{\kappa}$ while the hydrogen-like distribution is indicated by a cross. The location of the cross is placed at high $\tilde{\kappa}$ as this is indicative of its behavior.

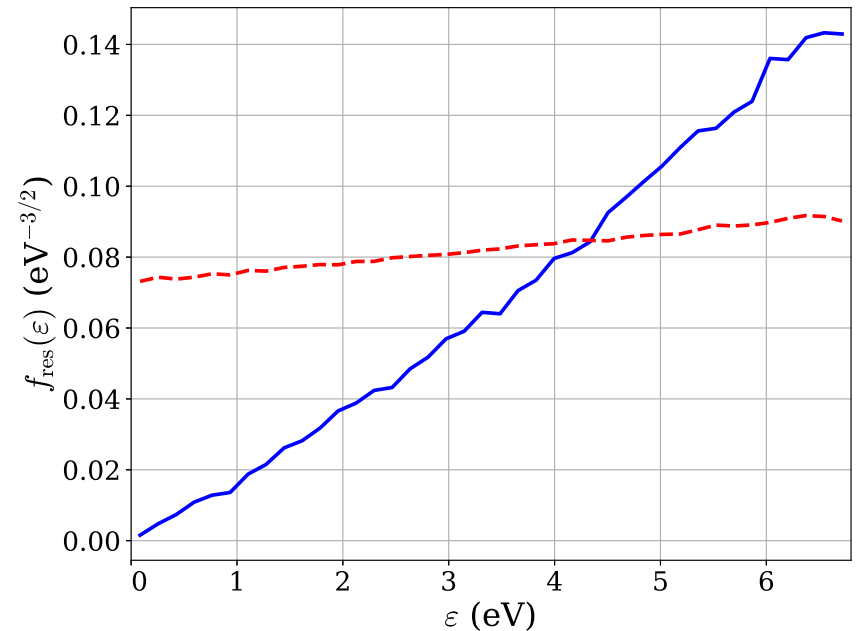

Figure 6. The distribution of positron energies immediately after a positron reaches energies below Ps formation for a distribution of $\tilde{\kappa}=1$ (blue solid) and a hydrogen-like $P_{K}$ distribution (red dashed). The distribution is normalized to $\int_{0}^{\varepsilon \mathrm{Ps}} d \varepsilon \sqrt{\varepsilon} f(\varepsilon)=1$.

high-energy behavior dictates the time to reach Ps formation energies, whereas the threshold regime influences the survival fraction of the positrons.

We note that our choice of $P_{K}$, fitted to a functional form for positron impact ionization (PII) of hydrogen given in Mikhailov et al. (2008b), differs from other studies. In Guessoum et al. (2005), Murphy (2005), and Bussard \& Ramaty (1978), an energy-loss distribution with a mean of $\varepsilon_{\mathrm{IP}} / 4$ and standard deviation of $2 \mathrm{eV}$ was used. This originated from a trend for the most probable speed of the ejected electron observed in the Born approximation for electron impact ionization (EII; Omidvar 1965). However, we note that the most probable energy, obtained by converting the speed distribution of Omidvar (1965) to an energy distribution, is actually $\varepsilon \rightarrow 0$. This corresponds to our observation of $\langle\kappa\rangle \rightarrow 1$.

Furthermore, near threshold energies the Born approximation is not valid, which is apparent from the disagreement with the experiment for the integrated cross section, both with
EII and PII (Mikhailov et al. 2008a, 2008b; Jones et al. 1993; Shah et al. 1987). In fact, the classical result for the integrated cross section in EII (Gryziński 1965) is closer to the experimental value, which was likely the motivation behind the choice of ionization cross section in Jean et al. (2009) and Alexis et al. (2014).

\section{Conclusion}

We have outlined a series of benchmarks for determining the positronium formation fraction in a collisional interstellar medium. These results show the need for a detailed description of the scattering dynamics and in particular the energy sharing fraction for ionization processes. The variation in all previous determinations of the Ps fraction can be explained by comparison to these benchmark examples. Benchmarking positron transport codes is critical in the interpretation of gamma telescope observations and, ultimately, for the understanding of the sources and sinks of positrons within the interstellar media. Future work exploring the details of thermalization in the interstellar media and the prospect of a low-energy reservoir of positrons is needed.

The sensitivity of the Ps formation fraction to the sharing fraction distribution, explored in this work, demonstrates the need for precise measurements and calculations of an energydifferentiated cross section for PII close to threshold. This is required for all constituents of the ISM so that accurate simulations of Ps formation fractions can be performed. We have also shown that the energy sharing distribution has significant effects on the low-energy reservoir of positrons and on their transit.

The authors would like to acknowledge Dr. Daniel Murtagh for helpful conversations in the initial stages of this work, in particular with regards to the low-energy reservoir and the excitation cross section. The authors would also like to thank Dr. Roland Crocker and Dr. Fiona Panther for useful discussions regarding ISM models and observational issues and Corey Hanrahan and Jonathon Liu for initial forays into the implementation of Monte Carlo simulations. 
J.R.M. and D.G.C. acknowledge support and funding from the Australian Research Council under Discovery Program grants DE160100098 and DE170101024.

\section{Appendix}

\section{Benchmark Values}

In this appendix we tabulate our numerical values for specific benchmark parameters so that other codes can be tested against our results. Tables 1, 2 and 3 correspond to benchmark sets $\mathrm{A}, \mathrm{B}$, and $\mathrm{C}$, respectively. In addition to the quantities discussed in the main text, we also include $n_{\mathrm{ion}}, n_{\mathrm{el}}$, and $n_{\mathrm{exc}}$, the average number of ionization, elastic, and excitation events, respectively, and $\langle\varepsilon\rangle_{\mathrm{Ps}}$, the average energy of the positron on forming Ps. See the text for the definition of the energy dependence of the cross sections. A constant value for $P(\varepsilon, \kappa)$ implies that $\tilde{\kappa}$ is equal to this value. All values given agree between two independent implementations to a relative error of $0.1 \%$. Note that $\tau_{\text {res }}^{*}=n_{0} \tau_{\text {res }}$.

Table 1

Quantities of Interest for the Benchmark Model A at Selected Parameters

\begin{tabular}{|c|c|c|c|c|c|c|c|c|c|c|c|}
\hline$A_{\text {ion }}$ & $\begin{array}{c}\lambda_{\text {ion }} \\
(\mathrm{eV})\end{array}$ & $P(\varepsilon, \kappa)$ & $\begin{array}{l}\varepsilon_{\mathrm{exc}} \\
(\mathrm{eV})\end{array}$ & $A_{\mathrm{exc}}$ & $\begin{array}{l}\lambda_{\text {exc }} \\
(\mathrm{eV})\end{array}$ & $\begin{array}{c}f_{\text {surv }} \\
(\%)\end{array}$ & $\left(10^{15} \stackrel{\left.\tau_{\text {res }}^{*} \mathrm{~m}^{-3}\right)}{\text { (n) }}\right.$ & $n_{\text {ion }}$ & $n_{\mathrm{el}}$ & $n_{\mathrm{exc}}$ & $\begin{array}{l}\langle\varepsilon\rangle_{\mathrm{Ps}} \\
(\mathrm{eV})\end{array}$ \\
\hline 0.3 & 30.0 & 1.0 & $\ldots$ & $\ldots$ & $\ldots$ & 0.512 & 231 & 725 & 35.6 & 0.0 & 52.7 \\
\hline 0.1 & 30.0 & 1.0 & $\ldots$ & $\ldots$ & $\ldots$ & 0.0172 & 678 & 710 & 102 & 0.0 & 70.8 \\
\hline 0.5 & 30.0 & 1.0 & $\ldots$ & $\ldots$ & $\ldots$ & 1.61 & 140 & 728 & 21.7 & 0.0 & 44.8 \\
\hline 1.0 & 30.0 & 1.0 & $\cdots$ & $\ldots$ & $\cdots$ & 5.35 & 70.0 & 731 & 11.2 & 0.0 & 35.3 \\
\hline 0.3 & 30.0 & 0.5 & $\ldots$ & $\ldots$ & $\ldots$ & 11.2 & 1.58 & 7.97 & 1.94 & 0.0 & 33.4 \\
\hline 0.3 & 30.0 & 0.8 & $\ldots$ & $\ldots$ & $\ldots$ & 1.86 & 4.18 & 21.0 & 4.18 & 0.0 & 41.3 \\
\hline 0.3 & 30.0 & 0.9 & $\ldots$ & $\ldots$ & $\ldots$ & 1.05 & 8.04 & 38.9 & 6.63 & 0.0 & 45.9 \\
\hline 0.3 & 30.0 & 0.95 & $\cdots$ & $\cdots$ & $\cdots$ & 0.747 & 14.9 & 68.3 & 9.8 & 0.0 & 48.9 \\
\hline 0.3 & 30.0 & 0.99 & $\ldots$ & $\ldots$ & $\ldots$ & 0.555 & 52.8 & 207 & 19.2 & 0.0 & 51.9 \\
\hline 0.3 & 30.0 & $P_{K}$ & $\cdots$ & $\cdots$ & $\cdots$ & 7.65 & 75.6 & 239 & 13.1 & 0.0 & 40.6 \\
\hline 0.3 & 10.0 & 1.0 & $\ldots$ & $\ldots$ & $\ldots$ & 0.493 & 674 & 711 & 95.8 & 0.0 & 62.6 \\
\hline 0.3 & 20.0 & 1.0 & $\ldots$ & $\ldots$ & $\ldots$ & 0.601 & 343 & 721 & 50.8 & 0.0 & 55.3 \\
\hline 0.3 & 40.0 & 1.0 & $\ldots$ & $\ldots$ & $\ldots$ & 0.398 & 175 & 726 & 28.0 & 0.0 & 51.7 \\
\hline
\end{tabular}

Table 2

Benchmark Values as Described in Table 1 but for the Benchmark Set B

\begin{tabular}{|c|c|c|c|c|c|c|c|c|c|c|c|}
\hline$A_{\text {ion }}$ & $\begin{array}{c}\lambda_{\text {ion }} \\
(\mathrm{eV})\end{array}$ & $P(\varepsilon, \kappa)$ & $\begin{array}{l}\varepsilon_{\mathrm{exc}} \\
(\mathrm{eV})\end{array}$ & $A_{\mathrm{exc}}$ & $\begin{array}{l}\lambda_{\operatorname{exc}} \\
(\mathrm{eV})\end{array}$ & $\begin{array}{r}f_{\text {surv }} \\
(\%)\end{array}$ & $\left(10^{15} \stackrel{\tau_{\text {res }}^{*}}{\mathrm{~s}} \mathrm{~m}^{-3}\right)$ & $n_{\text {ion }}$ & $n_{\mathrm{el}}$ & $n_{\mathrm{exc}}$ & $\begin{array}{l}\langle\varepsilon\rangle_{\mathrm{Ps}} \\
(\mathrm{eV})\end{array}$ \\
\hline 0.3 & 30.0 & 1.0 & 10.0 & 0.5 & 12.0 & 3.29 & 211 & 655 & 30.4 & 69.1 & 45.0 \\
\hline 0.3 & 30.0 & 1.0 & 10.0 & 0.1 & 12.0 & 0.872 & 227 & 709 & 34.4 & 15.3 & 50.8 \\
\hline 0.3 & 30.0 & 1.0 & 10.0 & 1.0 & 12.0 & 7.43 & 194 & 598 & 26.8 & 124 & 40.2 \\
\hline 0.3 & 30.0 & 1.0 & 10.0 & 1.5 & 12.0 & 11.8 & 180 & 551 & 24.0 & 169 & 36.9 \\
\hline 0.3 & 30.0 & 1.0 & 20.0 & 0.5 & 12.0 & 6.27 & 200 & 613 & 27.0 & 63.9 & 38.2 \\
\hline 0.3 & 30.0 & 1.0 & 30.0 & 0.5 & 12.0 & 7.78 & 190 & 578 & 24.3 & 59.5 & 33.6 \\
\hline 0.3 & 30.0 & 1.0 & 10.0 & 0.5 & 10.0 & 3.24 & 216 & 670 & 31.4 & 54.6 & 45.9 \\
\hline 0.3 & 30.0 & 1.0 & 10.0 & 0.5 & 20.0 & 3.11 & 192 & 592 & 26.8 & 128 & 42.5 \\
\hline 0.3 & 30.0 & $P_{K}$ & 10.0 & 0.5 & 12.0 & 10.8 & 73.4 & 230 & 12.2 & 25.9 & 37.2 \\
\hline 0.3 & 30.0 & $P_{K}$ & 10.0 & 0.1 & 12.0 & 8.2 & 75.2 & 237 & 12.9 & 5.42 & 39.8 \\
\hline 0.3 & 30.0 & $P_{K}$ & 10.0 & 1.0 & 12.0 & 14.4 & 71.1 & 222 & 11.4 & 49.1 & 34.6 \\
\hline 0.3 & 30.0 & $P_{K}$ & 10.0 & 1.5 & 12.0 & 17.9 & 69.1 & 215 & 10.7 & 70.2 & 32.6 \\
\hline 0.3 & 30.0 & $P_{K}$ & 20.0 & 0.5 & 12.0 & 11.8 & 71.9 & 224 & 11.4 & 25.0 & 33.3 \\
\hline 0.3 & 30.0 & $P_{K}$ & 30.0 & 0.5 & 12.0 & 12.6 & 70.4 & 218 & 10.7 & 24.2 & 30.8 \\
\hline 0.3 & 30.0 & $P_{K}$ & 10.0 & 0.5 & 10.0 & 11.0 & 73.9 & 232 & 12.3 & 20.2 & 37.6 \\
\hline 0.3 & 30.0 & $P_{K}$ & 10.0 & 0.5 & 20.0 & 10.1 & 70.8 & 221 & 11.5 & 50.5 & 36.1 \\
\hline
\end{tabular}


Table 3

Benchmark Values as Described in Table 1 but for the Benchmark Set C

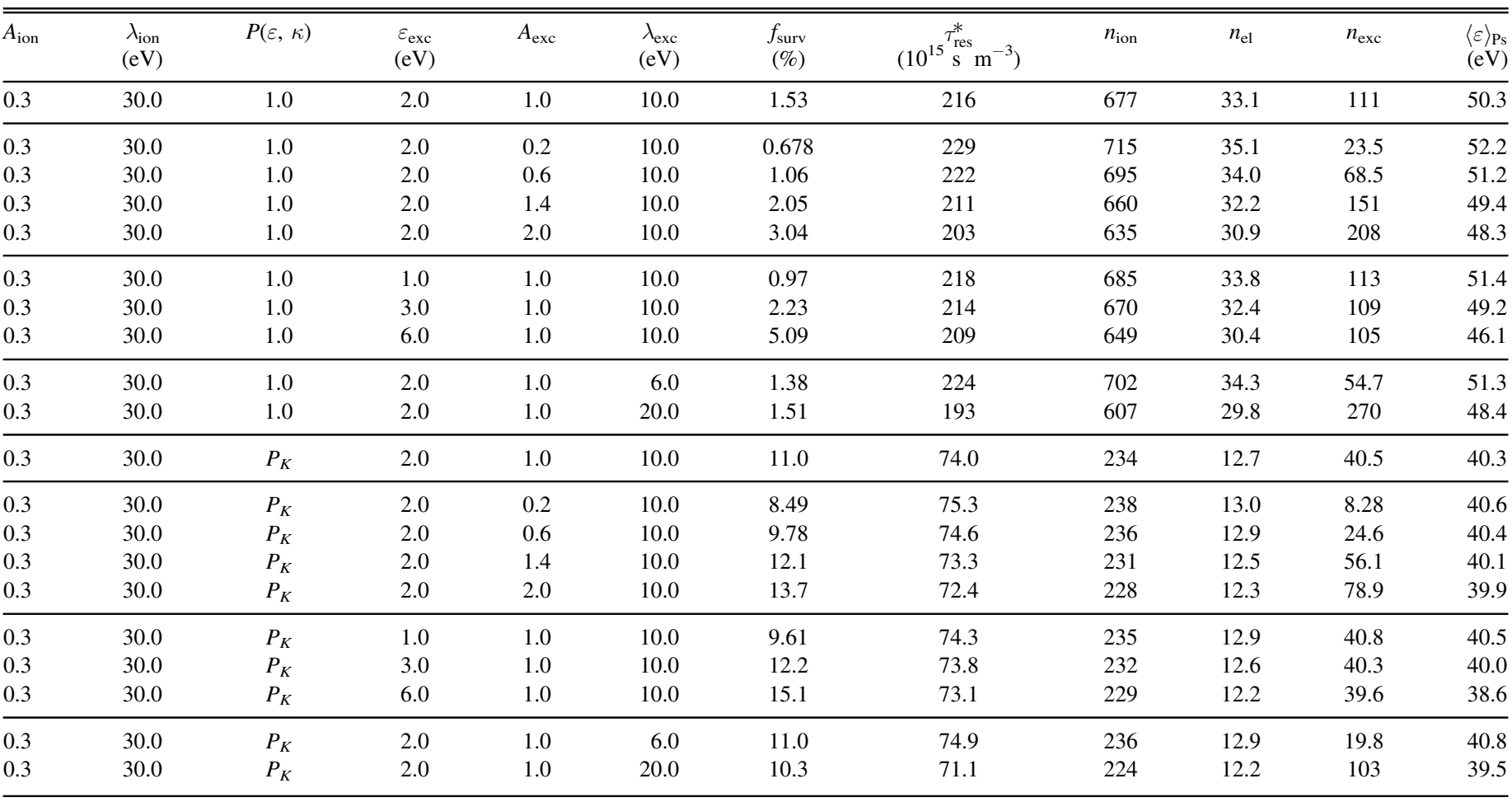

\section{ORCID iDs}

Daniel G. Cocks (i) https://orcid.org/0000-0002-9943-7100 Joshua R. Machacek (i) https://orcid.org/0000-00021404-7323

\section{References}

Alexis, A., Jean, P., Martin, P., \& Ferrière, K. 2014, A\&A, 564, A108 Bethe, H. 1930, AnP, 397, 325

Boyle, G. J., Tattersall, W. J., Cocks, D. G., McEachran, R. P., \& White, R. D. 2017, PSST, 26, 024007

Brown, B. L., Leventhal, M., Mills, A. P., \& Gidley, D. W. 1984, PhRvL, 53, 2347

Bussard, R., \& Ramaty, R. 1978, The Annihilation of Galactic Positrons, Tech. Rep. 79600, NASA

Charlton, M. 2009, JPhCS, 162, 012003

Churazov, E., Sazonov, S., Tsygankov, S., Sunyaev, R., \& Varshalovich, D. 2011, MNRAS, 411, 1727

Crocker, R. M., Ruiter, A. J., Seitenzahl, I. R., et al. 2017, NatAs, 1, 10

Danielson, J. R., Dubin, D. H., Greaves, R. G., \& Surko, C. M. 2015, RvMP, 87,247

Dujko, S., White, R. D., \& Petrovic, Z. L. 2008, JPhD, 41, 245205

Dujko, S., White, R. D., Petrović, Z. L., \& Robson, R. E. 2010, PhRvE, 81, 1 Gryziński, M. 1965, PhRvA, 138, A336

Guessoum, N., Jean, P., \& Gillard, W. 2005, A\&A, 436, 171

Higdon, J. C., Lingenfelter, R. E., \& Rothschild, R. E. 2009, ApJ, 698, 350 Jean, P., Gillard, W., Marcowith, A., \& Ferrière, K. 2009, A\&A, 508, 1099

Jones, G. O., Charlton, M., Slevin, J., et al. 1993, JPhB, 26, L483

Kernoghan, A. A., Robinson, D. J. R., McAlinden, M. T., \& Walters, H. R. J. 1996, JPhB, 29, 2089
Landau, L. D., \& Lifshitz, E. M. 1981, Quantum Mechanics: Non-Relativistic Theory (3rd edn; Oxford: Elsevier Butterworth-Heinemann)

Machacek, J. R., Blanco, F., Garcia, G., Buckman, S. J., \& Sullivan, J. P. 2016, $\mathrm{JPhB}, 49,0$

Marjanović, S., Banković, A., Cassidy, D., et al. 2016, JPhB, 49, 215001

Marjanović, S., Banković, A., White, R. D., et al. 2015, PSST, 24, 025016

Marjanović, S., Šuvakov, M., Engbrecht, J. J., \& Petrović, Z. L. 2012, NIMPB, 279,80

Martin, P., Strong, A. W., Jean, P., Alexis, A., \& Diehl, R. 2012, A\&A, 543, A3

Mikhailov, A., Nefiodov, A., \& Plunien, G. 2008a, PhLA, 372, 4451

Mikhailov, A., Nefiodov, A., \& Plunien, G. 2008b, PhLA, 372, 5171

Murphy, R. J. 2005, ApJS, 161, 495

Ness, K. F., \& Robson, R. E. 1986, PhRvA, 34, 2185

Omidvar, K. 1965, PhRv, 140, A26

Panther, F. H., Crocker, R. M., Birnboim, Y., Seitenzahl, I. R., \& Ruiter, A. J. 2018a, MNRAS, 474, L17

Panther, F. H., Seitenzahl, I. R., Crocker, R. M., et al. 2018b, PhRvD, 98 , 23015

Prantzos, N., Boehm, C., Bykov, A. M., et al. 2011, RvMP, 83, 1001

Raspopovic, Z. M., Sakadzic, S., Bzenic, S. A., \& Petrovic, Z. L. 1999, ITPS, 27, 1241

Reid, I. D. 1979, AuJPh, 32, 231

Robson, R., White, R., \& Hildebrandt, M. 2017, Fundamentals of Charged Particle Transport in Gases and Condensed Matter (London: CRC Press)

Shah, M. B., Elliott, D. S., \& Gilbody, H. B. 1987, JPhB, 20, 3501

Siegert, T., Diehl, R., Khachatryan, G., et al. 2016, A\&A, 586, A84

Tattersall, W. J., Cocks, D. G., Boyle, G. J., Buckman, S. J., \& White, R. D. 2015, PhRvE, 91, 43304

Wallyn, P., Durouchoux, P., Chapuis, C., \& Leventhal, M. 1994, ApJ, 422,610 